\title{
RADIOFREQUÊNCIA NÃO ABLATIVA NO TRATAMENTO DA INCONTINÊNCIA URINÁRIA DE ESFORÇO
}

\begin{abstract}
Andréa Queiroz Vilas Boas*, Cristina Aires Brasil**, Juliana Menezes Santos***, Luise de Souza Damasceno****, Maria Clara Neves Pavie Cardoso*****, Patrícia Lordêlo******,
\end{abstract}

Autor correspondente: Patrícia Lordelo - patricialordelo@bahiana.edu.br

* Médica ginecologista e obstetra, mestranda do curso de Tecnologia e Saúde pela Escola Bahiana de Medicina e Saúde Pública, ginecologista do Centro de Atenção ao Assoalho Pélvico, Salvador, Bahia, Brasil, andreaqvb@oi.com.br

** Fisioterapeuta graduada na Escola Bahiana de Medicina e Saúde Pública, fisioterapeuta do Centro de Atenção ao Assoalho Pélvico, Salvador, Bahia, Brasil, tinaabrasil@hotmail.com

*** Mestre em Tecnologia e Saúde pela Escola Bahiana de Medicina e Saúde Pública, Fisioterapeuta do Centro de Atenção ao Assoalho Pélvico, Salvador, Bahia Brasil, julianamenezes.fisio@gmail.com

**** Fisioterapeuta graduada na Escola Bahiana de Medicina e Saúde Pública, Fisioterapeuta do Centro de Atenção ao Assoalho Pélvico, Salvador, Bahia, Brasil, luisesaj10@hotmail.com

***** Fisioterapeuta graduada na Escola Bahiana de Medicina e Saúde Pública, Fisioterapeuta do Centro de Atenção ao Assoalho Pélvico, Salvador, Bahia, Brasil, mariaclarapavie@gmail.com

****** Doutorado em Medicina e Saúde Humana pela Escola Bahiana de Medicina e Saúde Pública, Professora Adjunta da Escola Bahiana de Medicina e Saúde Pública, coordenadora do Centro de Atenção ao Assoalho Pélvico, Salvador, Bahia Brasil, pvslordelo@hotmail.com

\section{Resumo}

A incontinência urinária de esforço(IUE) é definida como a queixa de perda urinária involuntária no esforço segundo o Consenso da Sociedade Internacional de Continência (International Continene Society - ICS). Ela chega a atingir $50 \%$ da população adulta feminina. Um dos mecanismos responsáveis por esse sintoma consiste na diminuição do colágeno nas paredes da uretra. A radiofrequência pode ser utilizada com o objetivo de promover a produção de colágeno. Assim a literatura tem colocado tanto o uso da radiofrequência ablativa vaginal como a não ablativa por via intra-uretral como possibilidades terapêuticas satisfatórias. A radiofrequência intra-uretral é uma técnica não ablativa porém desconfortável, de custo elevado principalmente pela necessidade de anestesia e de uso de antibiótico profilaxia, por ser aplicada dentro do canal uretral. No presente artigo serão descritas as teorias sobre a IUE, discutir sobre a radiofrequência aplicada com a finalidade terapêutica intra-uretral e fornecer uma perspectiva para a evolução da aplicação via vaginal, considerando que esta via aparece como uma alternativa menos invasiva e de baixo custo para o tratamento da IUE.

Palavras-chave: Incontinência Urinária por esforço; Radiofrequência; Terapêutica. 


\title{
NON-ABLATIVE RADIOFREQUENCY IN THE TREATMENT OF STRESS URINARY INCONTINENCE
}

\begin{abstract}
Stress urinary incontinence (SUI) is defined as the complaint of involuntary urine loss that happens whike the effort according to the International Continence Society (International Society continent to - ICS). It reaches up to $50 \%$ of the female adult population. One of the mechanisms responsible for this symptom consists in the reduction of collagen in the walls of the urethra. The radiofrequency can be used to stimulate the production of collagen. Some studies has placed the use of vaginal ablative radiofrequency and also the use of non-ablative radiofrequency through the urethra as satisfactory therapeutic possibilities. Althoug the intraurethral radiofrequency being a non-ablative technique, it's uncomfortable, and more expensive because in this technique it's necessary to use anesthesia and antibiotic prophylaxis because it happens into the urethral canal. On this article will be described theories about SUI, the use of radiofrequency as an intra-urethral way of treatment, and it will provide a perspective on the evolution of the application of vaginal radiofrequency therapeutic purpose, considering that it's less invasive and it's a low-cost alternative to SUI treatment.
\end{abstract}

Keywords: Urinary Incontinence Stress; Radiofrequency; Therapeutic.

\section{INTRODUÇÃO}

A incontinência urinária de esforço (IUE) é definida como a queixa de perda urinária involuntária no esforço, exercício, espirro ou tosse, segundo o consenso da Sociedade Internacional de Continência de 2009, que causa problema social e de higiene. A prevalência da IUE na população feminina adulta é de aproximadamente $50 \%$.(1) Apesar da sua alta prevalência, do sofrimento associado ao constrangimento pela perda de urina e ao impacto na qualidade de vida, muitas mulheres que experimentam sintomas de IUE não procuram tratamento ou permanecem sem resolução do quadro. Estima-se que em média somente uma em cada 4 mulheres procura auxilio por considerar os sintomas como característica fisiológica do envelhecimento. ${ }^{(2)}$ As opções terapêuticas não cirúrgicas para IUE incluem exercícios de fortalecimento do assoalho pélvico, treinamento com biofeedback, eletroestimulação funcional, dispositivos de aplicação intravaginais e modificações de comportamento. ${ }^{(3-6)}$ Existem opções de alta complexidade cirúrgica para a realização deste tratamento, sendo as mais utilizadas: tension free vaginal tape (TVT), tape and transobturador tape (TOT) e Marshal-Marchetti-Krants (MMK). ${ }^{(7-11)}$

As taxas de sucesso para as opções disponíveis não ultrapassam 50\% de resposta terapêutica com elevada taxa de retorno dos sintomas. ${ }^{(12,13)}$ Tem sido proposta a utilização da radiofrequência no tratamento da IUE, pois um dos fatores fisiopatogênicos desta incontinência é a diminuição de colágeno nas paredes da uretra, diminuindo os fatores que mantém a uretra fechada impedindo a perda de urina. Desta forma, serão descritas as teorias sobre a IUE, discutir sobre a radiofrequência aplicada com a finalidade terapêutica intra-uretral e fornecer uma perspectiva para a evolução da aplicação via vaginal, considerando que esta via aparece como uma 
alternativa menos invasiva e de baixo custo para o tratamento da IUE.

\section{FISIOPATOLOGIA DA IUE}

Ao longo dos anos surgiram várias teorias que tentaram explicar os mecanismos do surgimento da incontinência urinária de esforço, dentre esses os mais utilizados foram um relacionado a um inadequado suporte de sustentação da parede vaginal anterior e outra baseada numa possível alteração do mecanismo intrínseco da uretra. Por ser de origem multifatorial, ainda hoje falta um entendimento exato de como se processa o mecanismo da incontinência podendo-se afirmar que a etiologia desta patologia ainda se encontra indefinida. ${ }^{(14)}$ O complexo vesico-esfincteriano no sexo feminino encontra-se exposto a múltiplos fatores que podem estar relacionados a um maior risco de desenvolvimento da alteração, entre eles podemos citar os fatores que predispõe como a perda de colágeno, nutrição, obesidade, tabagismo, menopausa, constipação, medicações; envelhecimento, imobilidade física, gravidez, parto vaginal, cirurgias vaginais. Assim, considera-se que é a interação desses fatores em maior ou menor grau que acabam promovendo a lesão do mecanismo esfincteriano e surgimento da IUE. ${ }^{\left({ }^{14}\right)}$ Até a década de 70 prevaleceu a ideia que a continência urinária dependia da uretra estar localizada acima do assoalho pélvico para que a pressão transmitida para bexiga fosse igualmente transmitida para a uretra. ${ }^{(15)}$ Os estudos que utilizaram a urodinâmica para avaliar esta disfunção demonstraram que não somente a a localização da uretra tem uma influência nos fatores de continência mas também uma pressão de fechamento uretral e o comprimento da uretra funcional eram importantes para a manutenção deste mecanismo sem episódios de perdas. ${ }^{(15)}$ Em 1976 um novo conceito surgiu mostrando que alterações em ângulos uretrais e posicionamento uretral não explicavam todos os casos de IUE, surgindo o conceito de deficiência uretral intrínseca, onde foi demonstrado que a denervação do esfíncter uretral externo e da musculatura esquelética para-uretral, não exerciam influência na pressão de fechamento da uretra ao repouso ou na função do músculo liso uretral, nem todas as pacientes desenvolviam a IUE.(16) Em 1994 Delancey, trabalhou o conceito que combina a perda do suporte uretral e disfunção esfincteriana, confirmando a importância do músculo liso na manutenção da continência. ${ }^{(17,18)}$

\section{RADIOFREQUÊNCIA}

A RF é uma corrente de alta frequência, variando a frequência entre $30 \mathrm{KHz}$ e $3000 \mathrm{KHz}$ e vem sendo utilizada na Fisioterapia baseado no mecanismo de ação por uma produção de calor por conversão, este é um calor profundo, que atinge tecidos localizados a 0,5 centímetros de profundidade. ${ }^{(19,20)}$

A radiofrequência (RF) começou a ser utilizada com fins terapêuticos em 1920, quando foi introduzido o eletrocautério, ou seja, por um método ablativo. Em 1950 foi utilizada com o intuito de provocar lesões confiáveis nos sistema nervoso central, por neurocirurgiões. Em 1960 começou a utilização na área cardíaca. ${ }^{(21-24)}$

Atualmente a RF tem sido utilizada com diversos objetivos terapeuticos, dentre eles, podemos destacar o tratamento da dor, de neoplasias paraquimentosas e na ablação de feixes anômalos em casos de arritmias cardíacas. ${ }^{(25)}$

A passagem da corrente produz 3 fenômenos que resultam em aumento da temperatura, são eles: a vibração iônica, a rotação das moléculas dipolares e a distorção molecular. ${ }^{(26)}$

A vibração iônica é a forma mais eficiente de conversão de energia elétrica em calor. Os íons, que estão presentes em todos os tecidos do corpo, quando submetidos à RF vibram na mesma frequência, geram uma fricção e colisão, produzindo o aumento da temperatura. ${ }^{(26)}$

A rotação das moléculas dipolares é menos eficiente do que a vibração iônica. O corpo humano é formada, em sua maior totalidade, por água, que possue molécula eletricamente neutra em sua totalidade, 
que pode sofrer a conversão em dipolo quando submetida a ação de tração. Quando essa molécula é exposta a RF, ela gira de um lado ao outro produzindo colisão entre os tecidos adjacentes, produzindo conversão térmica. ${ }^{(20,26)}$

A distorção molecular gera uma conversão mínima de energia elétrica em calor. Acontece com as moléculas eletricamente neutras, que não tem movimento porque não tem carga elétrica, entretanto, os elétrons que rodeiam o núcleo são atraídos sofrendo uma distorção das suas órbitas. ${ }^{(20,26,27)}$

$\mathrm{O}$ efeito térmico produzido pela RF provoca uma desnaturação do colágeno promovendo uma contração imediata e efetiva das suas fibras, causando um processo inflamatório local e agudo, ativando os fibroblastos e, consequentemente, gerando uma neocolagenização e também proporciona uma reorgaização das fibras de colágeno. ${ }^{(26)}$

\section{RADIOFREQUENNCIA TRANSVAGINAL}

A fáscia endopélvica proporciona suporte para a região de colo da bexiga e uretra proximal. ${ }^{(28)} A$ RF transvaginal aplicada nessa região tem por objetivo gerar uma retração da sua membrana fibrosa que tem na sua composição o colágeno, como resultado ocorreria uma retração deste tecido proporcionando um aumentando da estabilidade do colo da bexiga e da uretra proximal. Dmochowski et all. (2003) em estudo prospectivo feito simultaneamente em 10 centros diferentes, aplicou a radiofrequência transvaginal em 120 mulheres com idade média de 49,9 anos que foram triadas de forma consecutiva e apresentavam em média um ou mais episódios de incontinência por dia. As pacientes foram avaliadas e todas apresentavam hipermobilidade vesical constatada pelo exame do swab ( swab média algodão mudar 38 graus). Foram excluídas do estudo aquelas que apresentaram instabilidade do detrusor após avaliação por cistometria. Os pacientes foram avaliados após 1 semana de pós-operatório e aos 3, 6 e 12 meses usando medidas objetivas e subjetivas. Dos 120 pacientes 96 completaram 1 ano de ava- liação. Tempo médio operatório foi menor do que 30 minutos, e todos os pacientes foram tratados em ambulatório. No pré-operatório $84 \%$ das pacientes apresentaram 1 ou mais episódios de incontinência urinária ao dia, após 3, 6, 12 meses de tratamento esse percentual caiu para $57 \%, 66 \%$ e $59 \%$ das pacientes relatando 1 ou nenhum episódio de incontinência. No seguimento de 12 meses 73\% das pacientes relataram estar continentes ou meIhorando e $76 \%$ das pacientes não apresentaram perda urinária durante a manobra de Valsalva em estudo urodinâmico. Num total, 30 casos foram classificados como falha do tratamento e $11 \mathrm{mu}$ Iheres foram perdidas durante o seguimento. Não ocorreram complicações no intra-operatório e $4 \%$ das pacientes tiveram pequenas complicações no pós operatório, dentre eles: sangramento vaginal devido a deiscência da incisão vaginal por 3 semanas, infecção do trato urinário por 1 mês e urgência miccicional durante 1 mês. Assim, a RF transvaginal aplicada na fáscia endopélvica demonstrou ser uma técnica segura e com resultados satisfatórios para o tratamento da incontinência urinária de esforço. ${ }^{(29)}$

\section{RADIOFREQUÊNCIA INTRAURTERAL}

A RF de aplicação transuretral tem o objetivo de reduzir e afunilar o colo da bexiga e a uretra proximal através da desnaturação do colágeno submucoso. Essa técnica foi utilizada de forma semelhante no tratamento da incontinência fecal e do refluxo gastresofágico. Difere da RF a ablativa por promover a desnaturação do colágeno e neocolanogênese, enquanto a outra gera necrose. ${ }^{(23,24)} \mathrm{A}$ aplicação é realizada por meio de uma sonda transuretral, $n^{\circ}$ 21, modelo especial de origem francesa, (Renessa Probe, NOVASYS Medical Inc.) que está conectado a um gerador de RF compacta disponível comercialmente (NOVASYS Medical Inc.). O sistema da radiofrequência transuretral é monopolar constituído de quatro eletrodos de agulha curvo de níquel-titanio com um calibre 23 no qual é introduzido na uretra por meio de uma sonda que após ser posicionada no interior do lúmen da bexiga é 
insuflada, semelhante a uma sonda de Foley. Através do eletrodo de agulha a energia é entregue em 9 posições com duração de 60 segundos, durante cada ciclo a submucosa é aquecida a $65^{\circ} \mathrm{C}$ produzindo a desnaturação do colágeno em 36 sítios em torno do colo da bexiga e da uretra proximal, sem que dano tecidual seja causado. Após a conclusão do último ciclo, o balão é desinsuflado e a agulha era retirada. ${ }^{(30)}$

Todo o tratamento incluído o posicionamento da paciente e aplicação da radiofrequência durou aproximadamente 20 minutos. Foram tratadas um total de 110 pacientes, 63 pacientes passaram pelo mesmo procedimento sem no entanto ocorrer a emissão da radiofrequência (grupo controle), a média de idade das pacientes foi de 50 anos. $O$ método demonstrou ser seguro e confortável. Este mecanismo de neocolanogenese tem como objetivo reduzir o comprimento regional do tecido dinâmico sem ocasionar uma restrição o redução do calibre lumial gerando assim uma mudança menos abrupta da anatomia da região. (23,24,31) $^{2}$

Para a aplicação da radiofrequência intrauretral faz-se necessário o uso de antibiótico via oral profilártico, sedativo via oral de 30 a 90 minutos antes do procedimento, além da aplicação de anestésico periuretal. o que torna o procedimento desconfortável e aumenta o risco de complicações.

Resposta clínica da Radiofrequência Intraurteral

Appell et al. (2006) em ensaio clínico randomizado com 173 mulheres, sendo 110 alocadas submetidas a RF intrauretral e 63 ao grupo sham, evidenciaram que $74 \%$ das mulheres com IUE de base moderada a grave foi verificado uma melhoria de 10 pontos ou mais no I-QOL após 12 meses da aplicação. As participantes do grupo submetido à RF demonstraram uma elevação do LPP (pressão de perda) em 12 meses, enquanto as do grupo controle apresentaram uma diminuição. (32)

Elser et al. (2009) realizaram um estudo prospectivo que teve como objetivo avaliar a eficácia na desnaturação do colágeno transuretral por meio da $\mathrm{RF}$, foram avaliados 136 objetivamente por meio do diário miccional e pad test, e uma avaliação subjetiva foi realizada por meio dos questionários I-QOL, UDI-6 e PGI-I. Foi observada que as pacientes apresentaram diminuição no número médio de perdas urinárias causas pelo esforço, $50 \%$ das participantes relataram uma diminuição de 50\% ou mais. Além disso foram observadas melhoras significativas no I-QOL e UDI-6. Quanto ao PGI $49,6 \%$ das participantes relataram estar melhor da queixa. Demonstrando uma alternativa viável para o tratamento da IUE. ${ }^{33)}$

A radiofrequência é uma técnica preconizada no tratamento para produção de colágeno cutâneo e intra-uretral, não apresentando rotineiramente reações adversas importantes, além de vermelhidão local ou leve edema, foram relatadas algumas complicações transitórias como disúria, infecção urinária, hematúria e disúria. ${ }^{(30,32,33)}$

\section{RADIOFREQUÊNCIA INTRAVAGINAL}

Millheiser LS et al. (2010), desenvolveu um estudo piloto com 24 mulheres pós parto vaginal com o objetivo de testar a tolerância e segurança da radiofrequência não ablativa em introito vaginal para minimizar a "frouxidão vaginal". Não ocorreu nenhum efeito adverso e não foi necessário o uso de anestésico. Um mês após a aplicação $67 \%$ da muIheres relataram melhora da frouxidão e 6 meses após $87 \%(p<0.001) .{ }^{(34)} \mathrm{Em} \mathrm{2013}$, Sekiguchi $Y$ et al., relacionaram a radiofrequência em introito vaginal com a função sexual demonstrando uma melhora significativa no escore do FSFI ( FEmale Sexual Function Index). ${ }^{(35)}$

\section{CONCLUSÃO}

Atualmente a radiofrequência por transferência capacitiva não ablativa vem sendo utilizada com sucesso via intrauretral, porém com riscos de alguns efeitos adversos como infecção urinária, disúria e hematúria. Refletindo o desejo maior das pacientes 
que é a busca de um tratamento minimamente invasivo, de preferencia não cirúrgico, seguro e rápido que leve a uma melhora na qualidade de vida, se faz necessário uma técnica que tenha uma menor probabilidade de acarretar os efeitos adversos da radiofrequência via intrauretral objetivando um tratamento com riscos minimizados, e um menor custo. Assim a radiofrequência vaginal surge como uma possibilidade terapêutica para a incontinência urinária de esforço.

\section{REFERÊNCIAS}

1. Norton P, Brubaker L. (2006) Urinary Incontinence in Women. Lancet; 367:56-57. 2006.

2. Yi SK, Cardozo L. Psychological morbidity and female urinary incontinence. Best Pract Res Clin Obstet Gynaecol. 2007;21(2):321-9. Epub 2007 Jan 3.

3. Latthe PM, Foon RM, Khan K. Nonsurgical treatment of stress urinary incontinence (SUI): Grading of evidence in systematic reviews. BJOG. 2008;115(4): 435-444.

4. Alewijnse D, Metsemakers JFM, Mesters IEPE, Van den Borne B. Effectiveness of pelvic floor muscle exercise therapy supplemented with a health education program to promote long-term adherence among women with urinary incontinence. Neurourol. Urodyn. 2003;22(4):284-295.

5. Janssen CCM, Lagro-Janssen ALM, Felling AJA. The effects of physiotherapy for female urinary incontinence: individual compared with group treatment. BJU International. 2001;87(3):201206.

6. Aukee P, Immonen P, Laaksonen DE, Laippala $P$, Penttinen J, Airaksinen $O$. The effect of home biofeedback training on stress incontinence. Acta Obstet Gynecol Scand. 2004;83(10):973977.

7. Brubaker L, Norton PA, Albo ME, Chai TC, Dandreo KJ, Lloyd KL et al. Adverse events over two years after retropubicor transobturator midurethral sling surgery: findings from the Trial of Midurethral Slings (TOMUS) study.
Am J Obstet Gynecol. 2001;205:498.el-6. doi:10.1016/j.ajog.2011.07.011.

8. Liapis A, Bakas P, Creatsas G. Burch colposuspension and tension-free vaginal tape in the management of stress urinary incontinence in women. Eur Urol. 2002;41:469-473.

9. Liapis A, Bakas P, Giner M, Creatsas G. Tension-free vaginal tape versus tension-free vaginal tape obturator in women with stress urinary incontinence. Gynecol Obstet Invest. 2006;62:160-164.

1O. Wang AC, Chen M-C. Comparison of tensionfree vaginal taping versus modified Burch colposuspension on urethral obstruc- tion: a randomized controlled. Neurourol Urodyn. 2003;22(3):185-90.

11. Albo ME, Richter HE, Brubaker L. Burch colpo suspension versus fascial sling to reduce urinary stress incontinence. N Engl J Med. 2007;356(21):2143-55.

12. Abrams P, Cardozo P, Fall M, Griffiths D, Rosier $P$, Ulmsten $U$ et al. The standardisation of terminology of lower urinary tract function: report from the standardisation sub-committee of the international continence society. Neurourol Urodyn. 2002;21(2):167-178.

13. Dillon B, Dmochowski R. Radiofrequency for the treatment of stress urinary incontinence in women. Curr Urol Rep. 2009;10(5):369-74.

14. Bump RC, Norton PA. Epidemiology and natural history of pelvic floor dysfunction. Obstet Gynecol Clin North Am. 1998;25:723-46.

15. Öbrink A, Bunne G, Ingelman-Sundberg A. Pressure transmission to the pre-urethral space in stress incontinence. Urol Res. 1978;6:135-140.

16. Mc Guire EJ. The efecct of sacral denervation on bladder and urethral function. Surg Gynecol Obstet. 1977;144:343-6.

17. DeLancey JO. The anatomy of the pelvic floor. Curr Opin Obstet Gynecol. 1994;6(4):313-6.

18. DeLancey JO. Structural support of the urethra as it relates to stress urinary incontinence: the hammock hypothesis. Am J Obstet Gynecol. 1994;170(6):1713-20; discussion 1720-3.

19. Lolis SM, Goldberg DJ. Radiofrequency in Cosmetic Dermatology: A review. Dermatolol Surg. 2012;38(11):1765-76. 
2O. Tecatherap - Vip: Manual de uso. Equipamiento professional y acessório para fisioterapia, rehabilitaction y medinica estética. VipElectromedicina

21. ECDJ, Luehring CC, Germano S. Estudo retrospectivo da eficácia e segurança da radiofrequência para flacidez e rugas faciais. Curitiba. 2010.

22. Paasch U, Bodendorf MO, Grunewald S, Simon JC. Skin rejuvenation by radiofrequency therapy: methods, effects and risks. J Dtsch Dermatol Ges. 2009;7(3):196-203.

23. Takahashi T, Garcia-Osogobio S, Valdovinos MA, Belmonte C, Barreto C, Velasco L. Extended two-year results of radio-frequency energy delivery for the treatment of fecalincon tinence(theseccaprocedure). Dis. colon rectum. 2003;46(6):711-715.

24. Lutfi RE, Torquati A, Richards WO, The endoscopic radiofrequency approach to management of GERD. Curr Opin Otolaryngol Head Neck Surg. 2004;12(3):191-196.

25. Almeida DB. Radiofrequência: conceitos técnicos e aplicações. Rev dor. 2007;8(4):11171121.

26. Carvalho GF, Silva RM, Mesquita Filho JJT, Meyer PF, Ronzio OA, Medeiros JO et al. Avaliação dos efeitos da radiofrequência no tecido conjuntivo. RBM. 2011; 68: 10-25.

27. Hodgkinson DJ. Clinical Applications of Radiofrequency: Nonsurgical Skin Tightening (Thermage). Clin Plast Surg. 2009;36(2):261-8.

28. Herschorn S. Female Pelvic Floor Anatomy: The Pelvic Floor, Supporting Structures, and Pelvic Organs. Rev Urol. 2OO4; 6(Suppl 5): S2-S1O.
29. Dmochowski RR, Avon M, Ross J, Cooper JM, Love RKB, Kohli $\mathrm{N}$ et al. Transvaginal radio frequency treatment of the endopelvic fascia: a prospective evaluation for the treatment of genuine stress urinary incontinence. The Journal of Urology. 2003;169:1028-1032.

3O. Elser D, Mitchell G, Miklos J, Nickell KG, Cline $\mathrm{K}$, Winkler $\mathrm{H}$ et al. Nonsurgical Transurethral Radiofrequency Collagen Denaturation: Results at Three Years after Treatment. Adv Urol. 2011.

31. Huang WC, Yang JM. Bladder neck funneling on ultrasound cystourethrography in primary stress urinary incontinence: a sign associated with urethral hypermobility and intrinsic sphincter deficiency. Urology. 2003;61(5):936-941.

32. Appell RA, Juma S, Wells WG, Lenihan JP, Klimberg IW, Kanellos A et al. Transurethral radiofrequency energy collagen microremodeling for the treatment of female stress urinary incontinence. Neurourol Urodyn. 2006;25(4):331-336.

33. Elser M, Mitchell GK, Miklos JK, Nickell KG, Cline K, Winkler $\mathrm{H}$ et al. Nonsurgical transurethral collagen denaturation for stress urinary incontinence in women: 12-month results from a prospective longterm study. J Minim Invasive Gynecol. 2009;16(1):56-62.

34. Millheiser LS, Pauls RN, Herbst SJ, Chen BH. Radiofrequency treatment of vaginal laxity after vaginal delivery: nonsurgical vaginal tightening. J Sex Med. 2010;7(9):3088-95.

35. Sekiguchi Y, Utsugisawa Y, Azekosi Y, Kinjo M, Song $M$, Kubota $Y$ et al. Laxity of the vaginal introitus after childbirth: nonsurgical outpatient procedure for vaginal tissue restoration and improved sexual satisfaction using low-energy radiofrequency thermal therapy. J Womens Health. 2013; 22(9):775-81. 